\title{
Tratamento da retinopatia diabética: percepções de pacientes em Rio Claro (SP) - Brasil
}

\author{
Treatment of diabetic retinopathy:patients'perceptions in Rio Claro \\ (São Paulo State)-Brazil
}

\author{
Valdir Balarin Silva ${ }^{1}$ \\ Edméa Rita Temporini ${ }^{2}$ \\ Djalma de Carvalho Moreira Filho ${ }^{3}$ \\ Newton Kara-José ${ }^{4}$
}

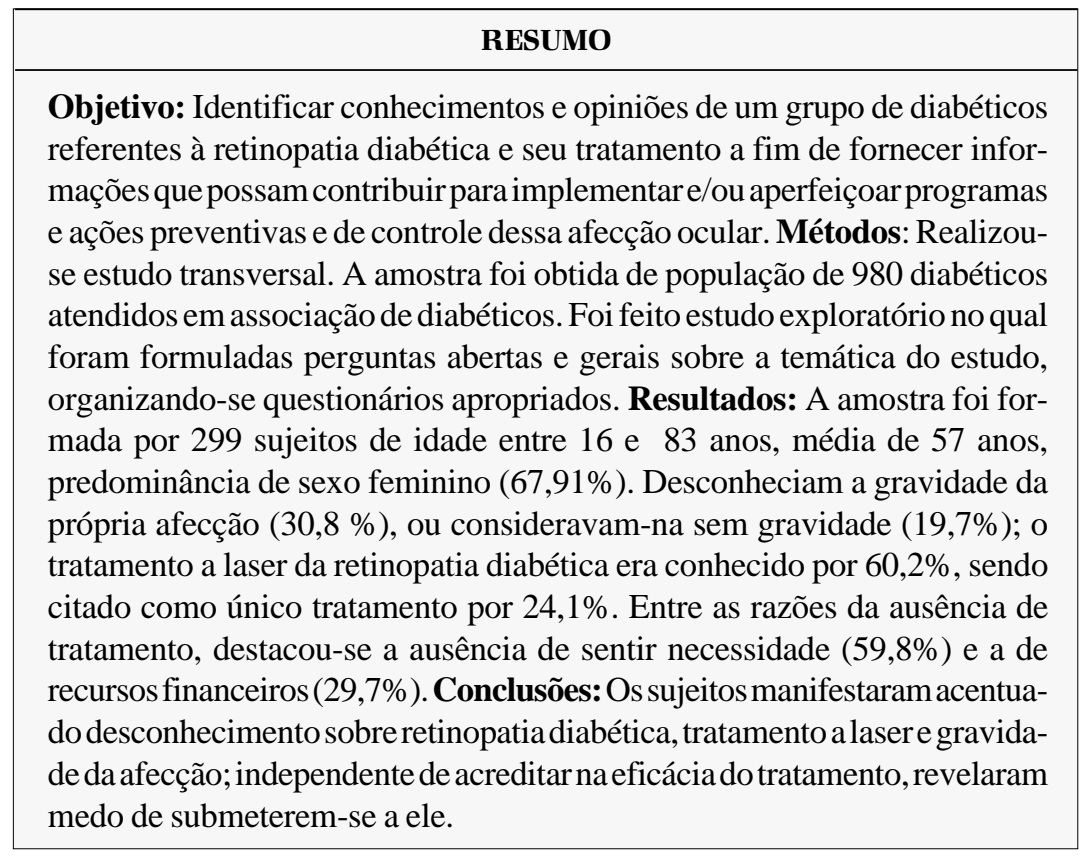

Descritores: Retinopatia diabética/tratamento; Retinopatia diabética/prevenção e controle; Saúde ocular; Diabetes mellitus; Saúde Pública; Percepção; Relações profissionalpaciente; Estudos transversais

Trabalho realizado no Departamento de Oftalmo-Otorrinolaringologia da Disciplina de Oftalmologia da Faculdade de Ciências Médicas da Universidade Estadual de Campinas (UNICAMP).

${ }^{1}$ Professor Assistente Doutor da Disciplina de Oftalmologia da Faculdade de Ciências Médicas da Universidade Estadual de Campinas (UNICAMP). Campinas (SP).

${ }^{2}$ Professor Livre Docente da Disciplina de Oftalmologia da Faculdade de Ciências Médicas da UNICAMP. Campinas (SP).

${ }_{3}^{3}$ Professor Livre Docente do Departamento de Medicina Preventiva e Social da Faculdade de Ciências Médicas da UNICAMP. Campinas (SP).

${ }^{4}$ Professor Titular da Disciplina de Oftalmologia da Faculdade de Ciências Médicas da UNICAMP. Campinas (SP).

Endereço para correspondência: Valdir Balarin Silva Rua 6 no 1353 - Rio Claro (SP) CEP 13500-190

E-mail: balarin@linkway.com.br

Recebido para publicação em 01.12.2003

Versão revisada recebida em 13.09.2004

Aprovação em 10.02.2005

Nota Editorial: Após concluída a análise do artigo sob sigilo editorial e com a anuência do Dr. João Carlos de Miranda Gonçalves sobre a divulgação de seu nome como revisor dele, agradecemos sua participação neste processo.

\section{INTRODUÇ̃̃̃OO}

O diabetes mellitus é a doença sistêmica mais freqüente como causa de cegueira nos Estados Unidos da América e no Reino Unido. Aproximadamente $10 \%$ da população cega nesses países é diabética. Entre os diabéticos a retinopatia diabética é a causa mais freqüente de cegueira, estando presente em mais de $70 \%$ desses $\operatorname{casos}^{(1)}$.

Em âmbito mundial a retinopatia diabética vem se tornando um problema de saúde pública. Após 15 anos de diabetes a prevalência de retinopatia entre os portadores de diabetes mellitus insulino-dependentes é de $97 \%$ e nos diabéticos não insulino-dependentes de $80 \%$.

Estimou-se que, em 1990, havia no Brasil cerca de 4,5 milhões de diabéti$\cos ^{(2)}$. Destes, 2 milhões desconheciam ser diabéticos e meio milhão não recebiam tratamento, o que permite concluir que diabetes mellitus é provavelmente a doença endócrino-metabólica mais importante no Brasil e seu impacto na saúde pública é comparável com o de países desenvolvidos, onde é considerado problema prioritário de solução.

Quase 50\% dos diabéticos entre 30 e 69 anos no Brasil desconheciam 
que estavam doentes (2), resultado comparável ao de outro estudo internacional ${ }^{(3)}$.

Dois estudos amplos e bem controlados, o "Diabetic Retinopathy Study" (D.R.S,) e o "Early Treatment Diabetic Retinopathy Study" (E.T.D.R.S.), demonstraram os benefícios da fotocoagulação a laser, que previne a perda visual progressiva mas não é capaz de reverter a acuidade visual já comprometida ${ }^{(4)}$.

Alguns autores ressaltam que freqüentemente, o paciente é encaminhado ou procura tratamento apenas em fases avançadas da retinopatia. No Brasil, várias pesquisas em diferentes grupos de pacientes diabéticos apontaram para situação simi$\operatorname{lar}^{(4-7)}$. Estas pesquisas entretanto não abordaram percepções de pacientes.

Quando a solução de problemas oftalmológicos depende da conduta das pessoas, a ação deve ser antecedida de pesquisa científica sobre o que as pessoas sabem, acreditam, desejam e fazem em relação à saúde ocular ${ }^{(8)}$.

Assim, acredita-se na importância de investigar fatores sociais relacionadas à prevenção e controle da retinopatia diabética, em grupo populacional afetado pelo diabetes mellitus.

Este estudo teve o objetivo de identificar conhecimentos e opiniões de um grupo de diabéticos referentes à retinopatia diabética e seu tratamento, a fim de fornecer informações que possam contribuir para implementar e/ou aperfeiçoar programas e ações preventivas e de controle dessa afecção ocular.

\section{MÉTODOS}

Realizou-se estudo transversal. A amostra foi obtida de população de diabéticos (980) atendidos em Associação de Diabéticos na época da pesquisa. Foram convidados por carta a dela participarem, esclarecendo-se os objetivos, data e local em que seria realizada. Essa carta foi entregue nos respectivos domicílios por meio de recrutas do exército na localidade. Obteve-se uma amostra não probabilística de tamanho 299 , formada pelos indivíduos que aceitaram participar do estudo.

$\mathrm{O}$ instrumento da pesquisa foi construído com base em estudo exploratório, no qual foram formuladas perguntas abertas e gerais sobre a temática do estudo. Realizaram-se entrevistas em profundidade com dez pessoas de ambos sexos, o que possibilitou obter conhecimentos que refletiam as características da realidade da população que se pretendia estudar, bem como o vocabulário comumente empregado na abordagem do diabetes ${ }^{(9)}$.

Complementando essas informações, utilizou-se como modelo um questionário empregado em pesquisa de percepções e conhecimentos sobre catarata, realizada no decorrer de projeto comunitário ${ }^{(9-10)}$.

Também serviram de base para elaboração do questionário sugestões de médicos endocrinologistas e a experiência de trabalho com pacientes portadores de diabetes e retinopatia diabética, na clínica oftalmológica privada e no serviço universitário. Foi realizado teste prévio para o aperfeiçoamento do instrumento. O questionário continha 30 questões (Anexo), das quais são apresentados os resultados referentes a 6 dessas questões. A análise do questionário completo foi discutida em tese de doutorado, apresentada em 2001(11).

A coleta de dados foi realizada mediante aplicação do questionário por entrevistas, houve divulgação prévia do projeto, pela imprensa escrita, falada e televisiva, fornecendo data, hora e local da realização dos exames, nessa divulgação à população era oferecido o exame de fundo de olho a todo paciente diabético, independente de pertencer à associação, mediante comprovação, por receita médica ou exame de glicemia.

Os sujeitos foram entrevistados por pessoal treinado, tendo-se assegurado o sigilo das informações. A coleta de dados foi realizada no período de 15 a 22/junho/1996. O tratamento estatístico dos dados foi realizado mediante o teste qui-quadrado, ao nível de significância de 0,05.

\section{RESULTADOS}

A amostra foi formada por 299 sujeitos de idades entre $16 \mathrm{e}$ 83 anos, com média de 57 anos, moda de 53 anos e predominância do sexo feminino $(67,9 \%)$ (Tabela 1$)$.

Entre os entrevistados desconheciam a gravidade da própria afecção ocular $(30,8 \%)$ ou consideravam-na sem gravidade $(19,7 \%)$. (Tabela 2$)$.

Declaram conhecer tratamento a laser $60,2 \%$ dos pacientes e, dos que conheciam, $24,1 \%$ citam-no como único tratamento disponível. Ressalta-se proporção apreciável dos entrevistados $(32,4 \%)$ declaram não saber da existência de cirurgia ou laser para tratamento de retinopatia diabética (Tabela 3).

Nota-se, na tabela 4, que a principal razão apontada para justificar a ausência de busca de tratamento foi a de não sentir necessidade $(59,8 \%)$.

A tabela 5 mostra, existir associação entre o medo de realizar o tratamento com laser e a opinião quanto à eficácia desse tratamento $(p \leq 0,0002)$. Excluindo a categoria "não sabe", permanece a associação ( $\mathrm{p} \leq 0,0039)$, indicando medo de realizar o tratamento com laser apesar de acreditar na eficácia do tratamento de retinopatia diabética.

\section{DISCUSSÃO}

A importância que as pessoas conferem à sua visão e aos cuidados para protegê-la depende, em grande parte, de padrões socioeconômicos, de conhecimentos, de hábitos e crenças aprendidos culturalmente ${ }^{(7)}$.

De outro lado nem sempre o fácil acesso a serviços de saúde constitui garantia para a obtenção de estados de higidez. Assim, o conhecimento da existência de serviços de saúde também não significa a busca intencional de assistência.

Entre os sujeitos deste estudo, aparentemente existe receio de tratamento com laser (Tabela 5). Este fato poderia explicar a procura tardia de tratamento com oftalmologista.

A prática diária com fotocoagulação e o contato com pacientes que dela necessitam mostra que muitos citam casos de 


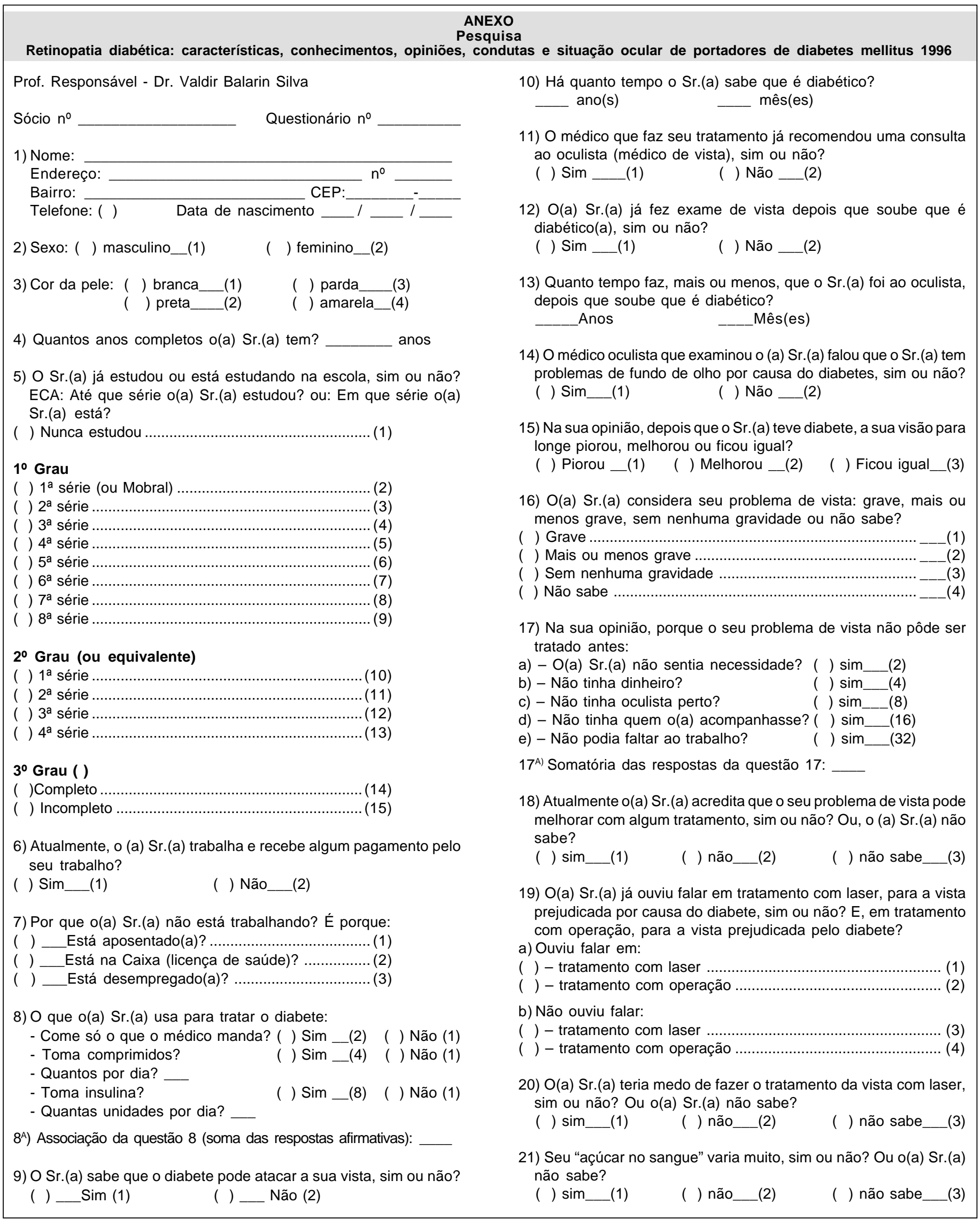




\section{ANEXO (Cont.)}

Pesquisa

Retinopatia diabética: características, conhecimentos, opiniões, condutas e situação ocular de portadores de diabetes mellitus 1996

22) $O($ a) Sr.(a) costuma ter:
a) pressão alta?
( ) Sim_(2) ( ) Não__(1)
b) adormecimento de mãos ou pés? ( ) Sim (4) ( ) Não- (1)
c) feridas nos pés? ( ) Sim_(8) ( ) Não__(1)
d) tem dor para urinar?
e) tem inchado?
f) a urina espuma muito?
( ) Sim_(16) ( ) Não__(1)
( ) Sim_(32) ( ) Não__(1)
( ) Sim_(64) ( ) Não__(1)

$\left.22^{A}\right)$ Somatória das respostas afirmativas da questão 22:
23) $\mathrm{O}$ (a) Sr.(a) faz diálise?
(1) $\quad$ Sim
(2)___Não
24) $\mathrm{O}$ (a) Sr.(a) sabe onde tratar vista prejudicada pelo diabete, sim ou não? ECA: qual é esse serviço?
( ) Não sei...
( ) Posto de saúde ...
( ) Convênio médico
( ) Médico particular
( ) Outros recursos

24^) Somatória das respostas afirmativas da questão "24":

25) Já tratou seu problema de fundo de olho com remédios, sim ou não? ECA: O que o(a) Sr.(a) usou?

( ) Não

( ) Sim. Quais?

Colírios
Pomada
Comprimido

( )

( )

( ).

$\left.25^{A}\right)$ Somatória das respostas afirmativas da questão "25":

26) Acuidade visual sem correção longe

O.D.

O.E.
27) Acuidade visual com correção longe

O.D. O.E.

28) Acuidade visual sem correção perto

O.D.

O.E.

29) Acuidade visual com correção perto

O.D._ O.E.

30) Teste de cores:
( ) Normal
(1)
( ) Alterado

Muito obrigado por sua cooperação.

Espaço reservado para o médico:

1) Presença de Retinopatia Diabética:

- Sim, proliferativa

- Sim, pré-proliferativa

- Não proliferativa

- Ausência de retinopatia

- Outros diagnósticos

( )__(1)

( )_(2)

( ) (3)

( ) (4)

Qual (is)? outros pacientes que, segundo eles, após iniciarem tratamento a laser, ficaram cegos.

Admite-se que após pantofotocoagulação pode ocorrer uma diminuição de acuidade visual que geralmente desaparece em algumas semanas. Entretanto, em 14\% dos casos há diminuição permanente de uma ou mais linhas de visão. Também nos tratamentos focais do edema macular, tardiamente pode ocorrer diminuição da acuidade visual por aumento da área de cicatriz do laser ou aparecimento de exsudatos duros lesando a fóvea.

O estudo epidemiológico de Wisconsin de retinopatia diabética (W.E.S.D.R.), realizado entre 1980 e 1982, mostrou que $55 \%$ dos pacientes com retinopatia proliferativa de alto risco estavam sem tratamento. Embora alguns deles possivelmente aguardavam tratamento, provavelmente existiam barreiras que ainda precisavam ser conhecidas ${ }^{(12)}$.

Proporção alta de entrevistados desconheciam a gravidade da própria afecção ou consideravam-na sem gravidade. Esse fato evidencia a necessidade de programa de prevenção de retinopatia, visando esclarecer a população a respeito da importância e utilidade das ações preventivas referentes à retinopatia diabética.

Embora o efeito benéfico do tratamento a laser na manutenção da visão seja bem conhecido, pouco se sabe sobre as respostas psicológicas ao tratamento. Muitas questões ainda ne- cessitam de investigação, tais como: a recomendação de tratamento precoce a laser não é seguida pelo paciente em virtude da ausência de sintomas visuais? Como o paciente responde às perdas de visão induzidas por tratamento a laser? ${ }^{(13)}$.

A principal razão apontada neste estudo para justificar a ausência de busca de tratamento foi a de não sentir necessidade $(59,8 \%)$. Resultado semelhante foi obtido por outro autor $^{(14)}$, evidenciando que cerca de $70 \%$ dos pacientes examinados declararam que não sabiam ter problemas com os olhos. Além desses, pode haver outros fatores, como falta de entendimento pelo paciente da necessidade do exame ou mesmo engajamento dos médicos ou pessoal paramédico em divulgar essa necessidade ${ }^{(15)}$.

A segunda razão mais mencionada foi "dificuldade financeira". Provavelmente essas pessoas não haviam sido informadas da existência de serviços públicos equipados para tratamento gratuito, e de acesso facilitado.

Proporção apreciável dos entrevistados declaram não saber da existência de cirurgia ou laser para tratamento da retinopatia diabética, apesar do convívio com outros diabéticos, médicos e informações veiculadas pela mídia.

Outro estudo mostrou que $95 \%$ dos médicos gerais declaravam recomendar exames anuais de fundo de olho a seus pacientes, entretanto apenas $43,4 \%$ dos pacientes confirma- 


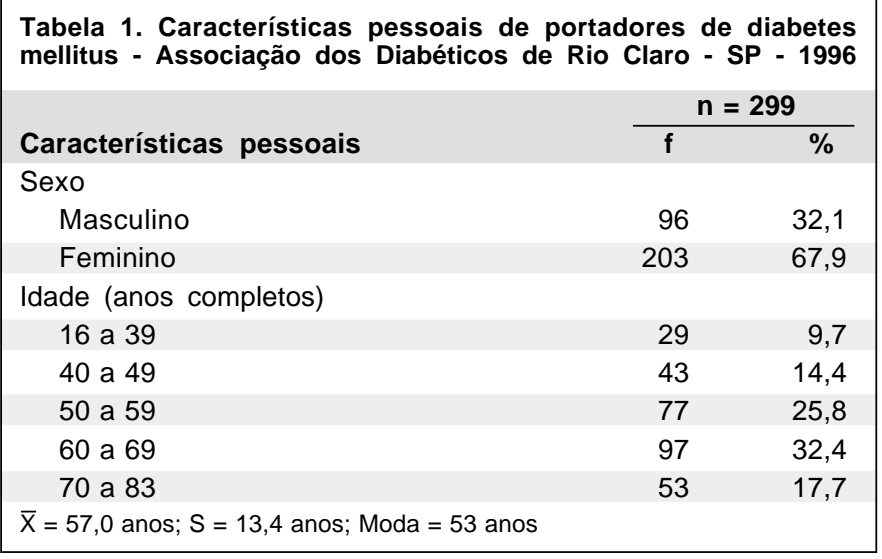

Tabela 2. Opinião sobre o grau de gravidade do próprio problema ocular. Portadores de diabetes mellitus participantes da Associação dos Diabéticos de Rio Claro - SP - 1996

\section{Auto-avaliação}

Sem nenhuma gravidade

Mais ou menos grave

Grave

Não sabe

Total

\begin{tabular}{rr}
\multicolumn{1}{c}{$\mathbf{f}$} & \% \\
59 & 19,7 \\
65 & 21,7 \\
83 & 27,8 \\
92 & 30,8 \\
299 & 100,0 \\
\hline
\end{tabular}

\begin{tabular}{|c|c|c|c|c|}
\hline \multicolumn{2}{|c|}{ Respondentes } & \multicolumn{3}{|c|}{ Tipos de tratamento (5) } \\
\hline$f$ & $\%$ & Cirurgia & Laser & Nenhum \\
\hline 108 & 36,1 & 36,1 & 36,1 & \\
\hline 97 & 32,4 & & & 32,4 \\
\hline 72 & 24,1 & & 24,1 & \\
\hline 22 & 7,4 & 7,4 & & \\
\hline 299 & 100,0 & 43,5 & 60,2 & 32,4 \\
\hline
\end{tabular}

ram essa recomendação e dos que procuraram oftalmologista, apenas $52,1 \%$ o faziam por iniciativa própria. Entre as razões dessas discrepância, os autores ressaltaram o diálogo insuficiente entre médico e paciente ${ }^{(16)}$. Como grupo, os clínicos gerais, endocrinologistas ou médicos de família, não estão preparados para o importante papel de primeiros triadores de saúde visual que o sistema atual de saúde requer. Isto não é surpresa, pelo fato de que poucos desses médicos receberam treino formal de oftalmologia como parte de sua educação médica ou programa de residência ${ }^{(17)}$.

A evolução dos cuidados com saúde deve ser acompanhada de reforma no processo de educação médica. No conceito de 'grupo cuidando do olho' deve-se incluir o médico geral, que freqüentemente será o primeiro profissional de saúde a examinar pacientes com distúrbios oculares. A participação de oftalmologistas na educação desses médicos é do maior interesse no desenvolvimento de atendimento de qualidade.

Existe tendência universal de aceitação dos critérios para referência e do algoritmo de tratamento proposto pela Academia Americana de Oftalmologia que propõe que todo diabético portador de D.M.N.I.D.(diabetes mellitus não insulino-dependente), tenha um exame de retina sob midríase já por ocasião do diagnóstico e depois anualmente. Os portadores de D.M.I.D. (diabetes mellitus insulino-dependente), teriam um exame oftalmológico cinco anos após o diagnostico e também anualmente. Antes da puberdade não é necessário o exame ${ }^{(15)}$.

O sentimento predominante de medo de tratamento reforça a idéia de que caberia aos serviços responsáveis por encaminharem a tratamento esclarecer que dificuldades iniciais são compensadas por manutenção por mais tempo de níveis adequados de visão e que a acuidade visual atual não é indício seguro de ausência de retinopatia ou seja, não assegura manutenção da visão por prazos mais longos.

Face aos resultados deste estudo, concluiu-se que os sujeitos manifestaram acentuado desconhecimento sobre retinopatia diabética, tratamento a laser e gravidade da afecção,

\begin{tabular}{|c|c|c|c|c|c|c|}
\hline \multirow{2}{*}{\multicolumn{2}{|c|}{ Respondentes }} & \multicolumn{4}{|c|}{ Razões (\%) } & \multirow{3}{*}{$\begin{array}{c}\text { Não tinha } \\
\text { acompanhante }\end{array}$} \\
\hline & & Não tinha & Não sentia & Não tinha & Não podia & \\
\hline 153 & 59,8 & & 59,8 & & & \\
\hline 76 & 29,7 & 29,7 & & & & \\
\hline 6 & 2,3 & 2,3 & 2,3 & & & \\
\hline 5 & 2,0 & & & 2,0 & & \\
\hline 4 & 1,5 & 1,5 & & 1,5 & & 1,5 \\
\hline 3 & 1,2 & & & & 1,2 & \\
\hline 3 & 1,2 & 1,2 & & & 1,2 & \\
\hline 2 & 0,7 & 0,7 & 0,7 & 0,7 & & 0,7 \\
\hline 1 & 0,4 & 0,4 & & & & 0,4 \\
\hline 1 & 0,4 & & & & & 0,4 \\
\hline 1 & 0,4 & 0,4 & & 0,4 & & \\
\hline 1 & 0,4 & 0,4 & 0,4 & 0,4 & & \\
\hline $256^{*}$ & 100,0 & 36,6 & 63,2 & 5,0 & 2,4 & 3,0 \\
\hline
\end{tabular}




\begin{tabular}{|c|c|c|c|c|c|c|}
\hline \multirow{3}{*}{$\begin{array}{l}\text { Medo do } \\
\text { tratamento }\end{array}$} & \multicolumn{6}{|c|}{ Eficácia do tratamento } \\
\hline & \multicolumn{2}{|c|}{ Acredita } & \multicolumn{2}{|c|}{ Não acredita } & \multicolumn{2}{|c|}{ Não sabe } \\
\hline & $f$ & $\%$ & $f$ & $\%$ & $f$ & $\%$ \\
\hline Tem medo & 50 & 24,6 & 2 & 6,6 & 7 & 10,6 \\
\hline Não tem medo & 111 & 54,6 & 22 & 73,4 & 30 & 45,4 \\
\hline Não sabe & 42 & 20,8 & 6 & 20,0 & 29 & 44,0 \\
\hline Total & 203 & 100,0 & 30 & 100,0 & 66 & 100,0 \\
\hline $\mathrm{p} \leq 0,0002$ & & & & & & \\
\hline
\end{tabular}

independente de acreditar na eficácia do tratamento, revelaram medo de submeterem-se a ele.

\section{ABSTRACT}

Purpose: To collect information and opinions from a group of diabetic patients regarding diabetic retinopathy and its treatment, in order to get reliable information that can help to improve programs and actions to control and prevent this ocular disease. Methods: A cross-sectional study was performed. The sample was from 980 diabetic patients seen in a diabetic association. A previous questionnaire was made with general questions about the main subject. Thereafter, an appropriate questionnaire was prepared. Results: The sample showed that among 299 patients with age ranging from 16 to 83 years, with a mean of 57 years, mainly female $(67.91 \%)$ did not know how severe their disease was $(30.8 \%)$, or believed that it was not a serious problem (19.7\%). The laser technique to solve diabetic retinopathy was known by $60.2 \%$ of the patients. It was reported as the only treatment available by $24.1 \%$. Among the reasons for no treatment $59.8 \%$ reported that they did not think it was necessary and $29.7 \%$ could not afford it. Conclusions: Patients showed lack of knowledge about how serious is diabetic retinopathy, the possibility of using laser technique for it and the severity of the disease. Some patients believed in the efficacy of the treatment and some patients did not, but all of them reported that they were afraid of submitting to it.
Keywords: Diabetic retinopathy/therapy; Diabetic retinopathy/prevention \&control; Eye health; Diabetes mellitus; $\mathrm{Pu}$ blic Health; Perception; Professional-patient relations; Crosssectional studies

\section{REFERÊNCIAS}

1. L'Esperance Jr FA, James Jr WA. Diabetic retinopathy clinical evaluation and management. St. Louis: Mosby; 1981. 294p.

2. Malerbi DA, Franco LJ. Multicenter study of the prevalence of diabetes mellitus and impaired glucose tolerance in the urban Brazilian population aged 30-69 yr. Diabetes Care. 1992;15(11):1509-16.

3. Harris MI, Hadden WC, Knowler WC, Bennett PH. Prevalence of diabetes and impaired glucose tolerance and plasma glucose levels in U.S. population aged 20 -74 years. Diabetes. 1987;36(4):523-34.

4. Arrata KM, Waszak DMJ, Delai NR, Moreira Jr CA. Detecção precoce da retinopatia diabética: análise de 296 pacientes em um hospital universitário. Rev Bras Oftalmol. 1996;55(1):63-9.

5. Foss MC, Paccola GM, Souza NV, Iazigi N. Estudo analítico de uma amostra populacional de diabéticos tipo II da região de Ribeirão Preto (SP). AMB Rev Assoc Med Bras. 1989;35(5):179-83.

6. Casella AMB, Bonomo PP, Farah ME. Prevalência da retinopatia diabética em pacientes diabéticos, atendidos pelo Sistema Único de Saúde (SUS) de Londrina - Paraná. Arq Bras Oftalmol. 1994;57(1):5-9.

7. Temporini ER. Pesquisa de oftalmologia em saúde pública: considerações metodológicas sobre fatores humanos. Arq Bras Oftalmol. 1991,54(6):279-81.

8. Piovesan A, Temporini ER. Pesquisa exploratória: procedimento para o estudo de fatores humanos no campo da saúde pública. Rev Saúde Pública. 1995; 29(4):318-25

9. Temporini ER, Kara-José N, Kara-José Jr N. Catarata senil: características e percepções de pacientes atendidos em projeto comunitário de reabilitação visual. Arq Bras Oftalmol. 1997;60(1):79-83.

10. Klein R, Klein BE, Moss SE, Davis MD, DeMets DL. The Wisconsin epidemiologic study of diabetic retinopathy. VI Retinal photocoagulation. Ophthalmology. 1987;94(7):747-53.

11. Silva VB. Retinopatia diabética: Características, conhecimentos, opiniões, condutas e situação ocular de portadores de diabete mellitus [tese]. Campinas: Universidade Estadual de Campinas; 2001. 125p.

12. Wulsin LR, Jacobson AM, Rand LI. Psychosocial aspects of diabetic retinopathy. Diabetes Care. 1987;10(3):367-73.

13. Moss SE, Klein R, Klein BE. Factors associated with having eye examinations in persons with diabetes. Arch Fam Med. 1995;4(6):529-34.

14. Kozousek W, Broun M G, Cottle R, Hicks VA, Langille DB, Dingle J. Use of ophthalmic services by patients in Nova Scotia. Can J Ophthalmol. 1993; 28(1):7-10. Comment in: Can J Ophthalmol. 1993;28(1):3-6.

15. Screening guidelines for diabetic retinopathy. American College Of Physicians, American Diabetes Association And American Academy Of Ophthalmology. Ann Int Med. 1992;116(8):683-5.

16. Kraft SK, Marrero DG, Lazaridis EN, Fineberg N, Qiu C, Clark CM. Primary care physician's practice patterns and diabetic retinopathy. Currente levels of care. Arch Fam Med. 1997;6(1):29-37. Comment in: Arch Fam Med. 1997; 6(1):38-41.

17. Lawler FH, Viviani N, Patient and physician perspectives regarding treatment of diabetes: compliance with practice guidelines. J Fam Pract. 1997;4494):369-73. 